\title{
D-Dimer elevation and adverse outcomes
}

\author{
Rim Halaby • Christopher J. Popma • Ander Cohen • Gerald Chi • \\ Marcelo Rodrigues Zacarkim - Gonzalo Romero - Samuel Z. Goldhaber • \\ Russell Hull • Adrian Hernandez • Robert Mentz $\cdot$ Robert Harrington • \\ Gregory Lip - Frank Peacock • James Welker • Ignacio Martin-Loeches • \\ Yazan Daaboul · Serge Korjian · C. Michael Gibson
}

Published online: 9 July 2014

(C) The Author(s) 2014. This article is published with open access at Springerlink.com

\begin{abstract}
D-Dimer is a biomarker of fibrin formation and degradation. While a D-dimer within normal limits is used to rule out the diagnosis of deep venous thrombosis and pulmonary embolism among patients with a low clinical probability of venous thromboembolism (VTE), the prognostic association of an elevated D-dimer with adverse outcomes has received far less emphasis. An elevated Ddimer is independently associated with an increased risk for incident VTE, recurrent VTE, and mortality. An elevated D-dimer is an independent correlate of increased mortality and subsequent VTE across a broad variety of disease states. Therefore, medically ill subjects in whom the D-dimer is elevated constitute a high risk subgroup in which the prospective evaluation of the efficacy and safety of antithrombotic therapy is warranted.
\end{abstract}

R. Halaby · C. J. Popma · G. Chi · M. R. Zacarkim · G. Romero ·

Y. Daaboul · S. Korjian · C. M. Gibson $(\bowtie)$

Cardiovascular Division, Department of Medicine, Beth Israel

Deaconess Medical Center, Harvard Medical School, East

Campus, RW 459, Boston, MA 02215, USA

e-mail: charlesmichaelgibson@gmail.com

A. Cohen

King's College Hospital, London, UK

S. Z. Goldhaber

Cardiovascular Division, Department of Medicine, Brigham and

Women's Hospital, Harvard Medical School, Boston, MA, USA

R. Hull

Department of Medicine Calgary, Foothills Hospital, Calgary, Canada

A. Hernandez $\cdot$ R. Mentz

Division of Cardiology, Department of Medicine, Duke

University Medical Center, Durham, NC, USA
Keywords D-Dimer - Deep vein thrombosis · Mortality · Prognosis $\cdot$ Pulmonary embolism
Abbreviations
VTE Venous thromboembolism
PE Pulmonary embolism
DVT Deep vein thrombosis

\section{Introduction}

D-Dimer is a biomarker of fibrin formation and degradation [1-3]. It may be elevated not only in patients with acute thrombosis, but also among the elderly as well as in a variety of illnesses $[4,5]$. While a D-dimer within normal limits is used to rule out the diagnosis of deep venous

\author{
R. Harrington \\ Department of Medicine, Stanford University, Stanford, CA, \\ USA \\ G. Lip \\ Centre for Cardiovascular Sciences, City Hospital, University \\ of Birmingham, Birmingham, UK \\ F. Peacock \\ Emergency Department, Baylor College of Medicine, Houston, \\ TX, USA \\ J. Welker \\ Anne Arundel Medical Center, Anne Arundel Health System \\ Research Institute, University of Maryland School of Medicine, \\ Baltimore, MD, USA
}

I. Martin-Loeches

Critical Care Centre, Corporació Sanitària I Universitària Parc

Taulí Hospital De Sabadell Parc Taulí, Barcelona, Spain 
thrombosis and pulmonary embolism among patients with a low clinical probability of venous thromboembolism [6, 7], the prognostic association of an elevated D-dimer with adverse outcomes has received far less emphasis.

\section{Methods}

A search of the Pubmed and Cochrane databases was independently performed by four individuals. The keyword used for the search was D-dimer in combination with other words such as prognosis, mortality, recurrence, incidence, and occurrence. The search was not limited by country or language and it included prospective and retrospective observational studies, clinical trials and systematic reviews. Study selection was initially based on the review of the title and it yielded a total of 87 articles. The articles were categorized as follows (some articles fit more than one category): D-dimer and mortality, D-dimer and occurrence of VTE, and D-dimer and recurrence of VTE. The four individuals reviewed the articles for quality assessment and extracted information about the study population, study design, statistical analysis, outcomes (symptomatic DVT, asymptomatic DVT, PE, VTE and mortality), results and limitations. Based on their methodology, adequacy of their sample size, and statistical analysis, a total of 37 articles were considered of at least average quality and were included in this manuscript.

\section{D-Dimer and subsequent occurrence of VTE}

Among acutely and critically ill patients, an elevated Ddimer is associated with an increased risk of a subsequent occurrence of a first episode of VTE independent of age, sex, race, body mass index, and medical illnesses [8-12]. In fact, among 7581 acutely ill medical patients enrolled in the MAGELLAN study, the incidence of a subsequent VTE was 3.5 times higher in patients with baseline concentrations of D-dimer equal or greater than two times the upper limit of normal compared to those with normal Ddimer (OR 2.29; $95 \%$ CI 1.75-2.98) [12]. In the general population, an elevated D-dimer is associated with an increased risk of a subsequent first episode of VTE among 923 subjects during 8 years of follow-up [13].

In various disease states, D-dimer is associated with the subsequent first occurrence of VTE and adverse clinical outcomes. During ischemic stroke, there is activation of the coagulation and fibrinolytic systems which may be reflected by elevations in D-dimer $[14,15]$. In a prospective multi-center study including 1,380 stroke patients followed for 12 months, an elevated D-dimer measured at the time of hospitalization was independently associated with the subsequent first occurrence of VTE (OR 3.45; $95 \% \mathrm{CI}$ 2.01-8.52) [15].

As a specific pathologic state, cancer is associated with a higher risk of subsequent first occurrence of VTE, a risk further amplified by hospitalization, surgical intervention, or chemotherapy [16]. D-Dimer is significantly higher among cancer patients who subsequently develop a first episode of VTE compared to those who do not [17, 18]. Among 821 cancer patients with a twofold increase in Ddimer concentration, there was a 1.3 fold increase in the first occurrence of VTE both in univariate (95\% CI $1.1-1.5 ; p<0.001)$ and multivariate analyses (HR 1.3; $95 \%$ CI 1.2-1.6; $p<0.001$ ) following adjustment for age, sex, radiotherapy, chemotherapy, and surgery [17]. The association of an elevated D-dimer with the first occurrence of VTE has been validated in multiple studies among patients with a variety of oncologic pathologies including colorectal, lung, and gynecological cancer [19-23].

\section{D-Dimer and recurrence of VTE}

While the previous studies evaluated the association of $\mathrm{D}$ dimer with the first occurrence of VTE, recurrent VTE following an initial episode is associated with an elevated D-dimer measured 3 months following discontinuation of anticoagulation therapy [24, 25]. The association of Ddimer and VTE recurrence is significant among both noncarriers (HR 2.43; $95 \%$ CI 1.18-4.61) and carriers of thrombophilia (HR 8.34; $95 \%$ CI 2.72-17.43) [26]. In a systematic review of seven studies involving 1888 patients with a first unprovoked episode of VTE, those with an elevated D-dimer measured following withdrawal of anticoagulation therapy had an annual rate of VTE recurrence of $8.9 \%$ compared to $3.5 \%$ among patients with a normal D-dimer [27]. In a separate patient level meta-analysis of the same population, the annual rates of VTE recurrence were 8.8 and $3.7 \%$ for patients with elevated and normal D-dimer, respectively [28].

\section{D-Dimer and mortality}

An elevated D-dimer is associated with short and long term increased mortality [12, 29]. In a meta-analysis of five studies $(n=2,885)$ of patients with pulmonary embolism, an elevated D-dimer was associated with increased all cause 30 day mortality (OR 2.76; $95 \%$ CI 1.83-4.14). Furthermore, in a 4 study meta-analysis $(\mathrm{n}=1,254)$ an elevated D-dimer was associated with a higher 3-month mortality (OR 4.29; $95 \%$ CI 1.70-10.79) [29]. 
The prognostic significance of D-dimer among stroke patients is mixed. Although one study did not find an association between D-dimer and mortality [30], others reported that $\mathrm{D}$-dimer is elevated among stroke patients who suffer a subsequent vascular death $(p=0.0201)$ [31]. Furthermore, an elevated D-dimer within the first weeks of ischemic stroke is associated with poorer survival independent of age, stroke type, or severity (HR 6.43; $95 \%$ CI 2.83-14.62; $p<0.0001$ ) [32].

Among cancer patients, D-dimer may be elevated not only in the presence of documented VTE but also in the absence of any detectable thrombus [33]. Elevated D-dimer is associated with an increased risk of mortality in a variety of cancers independent of age, sex, documented VTE, and types of malignancy $[17,33,34]$. In a meta-analysis of 13 studies of lung cancer patients in particular, elevation of Ddimer was associated with increased mortality (HR 2.06; $95 \%$ CI 1.64-2.58; $p=0.0001$ ) [35].

Like cancer, D-dimer is associated with higher mortality among patients with cardiovascular disease. In a study of 174 patients with heart failure, a D-dimer $>1,435 \mathrm{ng} / \mathrm{mL}$ was associated with increased cardiovascular mortality (HR 3.25; $95 \%$ CI 1.647-6.414; $p=0.001$ ) [36]. Moreover, in the AtheroGene Study of 1,057 patients with coronary artery disease prospectively followed for a median of 6.6 years, an elevated D-dimer was independently associated with high cardiovascular mortality [37]. Among patients with aortic dissection, an elevated D-dimer is associated with increased hospital mortality [38, 39].

In the setting of community acquired pneumonia and sepsis, elevation in D-dimer is independently associated with increased mortality [40-42]. Finally, even in the absence of clinically overt disease, among 17,359 subjects free of cardiovascular disease and cancer from the MOLI-SANI prospective cohort study, an elevated D-dimer was associated with increased mortality over the subsequent 4 years [43].

\section{Limitations}

Although multiple studies have demonstrated an association between elevated D-dimer and prognosis, no single cutpoint has been identified which consistently optimizes the prognostic value of the biomarker. The cut-point evaluated in multiple studies is that which exceeds the conventional D-dimer cut-off value ( $500 \mathrm{ng} / \mathrm{mL})$. However, the threshold for D-dimer exceeded $5,500 \mathrm{ng} / \mathrm{mL}$ in one study [44]. D-Dimer concentrations were measured only once in the majority of the studies.

Comparing clinical outcomes between studies which utilized different D-dimer assays and cut-points is challenging. D-dimer assays are not standardized, and some assays express D-dimer in fibrin equivalent units (FEU), while others express it in D-dimer units (one D-dimer unit is approximately two FEU) [33]. Moreover, D-dimer is also influenced by age [45] and concomitant medical conditions, such as atrial fibrillation, heart failure, peripheral artery disease and renal failure [36, 46-49]. The elevation in D-dimer in these patient groups is a limitation for both the diagnostic and prognostic role of D-dimer, particularly among the elderly and patients with renal failure among whom prolonged VTE prophylaxis can be problematic due to excess inadvertent bleeding $[50,51]$.

Finally, the outcomes of the studies regarding the occurrence of VTE events were not consistent across different studies as some included asymptomatic DVT while others did not. In addition, not all studies ruled out the presence of asymptomatic thrombus at the time of D-dimer measurement, and the possibility that the subsequent VTE episode was due to an existing clot cannot be excluded.

\section{Conclusion}

D-Dimer elevation is associated with the risk of first VTE occurrence, VTE recurrence, and mortality. An elevated Ddimer is an independent correlate of increased mortality and subsequent VTE across a broad variety of disease states. Therefore, medically ill subjects in whom the Ddimer is elevated constitute a high risk subgroup in which the prospective evaluation of the efficacy and safety of antithrombotic therapy is warranted.

Open Access This article is distributed under the terms of the Creative Commons Attribution License which permits any use, distribution, and reproduction in any medium, provided the original author(s) and the source are credited.

\section{References}

1. Adam SS, Key NS, Greenberg CS (2009) D-Dimer antigen: current concepts and future prospects. Blood 113(13):2878-2887

2. Ariens RA, de Lange M, Snieder H, Boothby M, Spector TD, Grant PJ (2002) Activation markers of coagulation and fibrinolysis in twins: heritability of the prethrombotic state. Lancet 359(9307):667-671

3. Rosendaal FR (1999) Venous thrombosis: a multicausal disease. Lancet 353(9159):1167-1173

4. Douma RA, le Gal G, Sohne M, Righini M, Kamphuisen PW, Perrier A et al (2010) Potential of an age adjusted D-dimer cut-off value to improve the exclusion of pulmonary embolism in older patients: a retrospective analysis of three large cohorts. BMJ (Clinical research ed) 340:c1475

5. Heit JA, Silverstein MD, Mohr DN, Petterson TM, O'Fallon WM, Melton LJ 3rd (2000) Risk factors for deep vein thrombosis and pulmonary embolism: a population-based case-control study. Arch Intern Med 160(6):809-815 
6. Wells PS, Owen C, Doucette S, Fergusson D, Tran H (2006) Does this patient have deep vein thrombosis? JAMA 295(2):199-207

7. Wells PS, Anderson DR, Rodger M, Stiell I, Dreyer JF, Barnes D et al (2001) Excluding pulmonary embolism at the bedside without diagnostic imaging: management of patients with suspected pulmonary embolism presenting to the emergency department by using a simple clinical model and D-dimer. Ann Intern Med 135(2):98-107

8. Kollef MH, Eisenberg PR, Shannon W (1998) A rapid assay for the detection of circulating D-dimer is associated with clinical outcomes among critically ill patients. Crit Care Med 26(6):10541060

9. Shorr AF, Trotta RF, Alkins SA, Hanzel GS, Diehl LF (1999) DDimer assay predicts mortality in critically ill patients without disseminated intravascular coagulation or venous thromboembolic disease. Intensive Care Med 25(2):207-210

10. Fan J, Li X, Cheng Y, Yao C, Zhong N (2011) Measurement of Ddimer as aid in risk evaluation of VTE in elderly patients hospitalized for acute illness: a prospective, multicenter study in China. Clin Invest Med 34(2):E96-E104

11. Desjardins L, Bara L, Boutitie F, Samama MM, Cohen AT, Combe $S$ et al (2004) Correlation of plasma coagulation parameters with thromboprophylaxis, patient characteristics, and outcome in the MEDENOX study. Arch Pathol Lab Med 128(5):519-526

12. Cohen AT, Spiro TE, Spyropoulos AC, Desanctis YH, Homering M, Buller HR et al (2014) D-Dimer as a predictor of venous thromboembolism in acutely ill, hospitalized patients: a subanalysis of the randomized controlled MAGELLAN trial. J Thromb Haemost 12(4):479-487

13. Cushman M, Folsom AR, Wang L, Aleksic N, Rosamond WD, Tracy RP et al (2003) Fibrin fragment D-dimer and the risk of future venous thrombosis. Blood 101(4):1243-1248

14. Tohgi H, Kawashima M, Tamura K, Suzuki H (1990) Coagulation-fibrinolysis abnormalities in acute and chronic phases of cerebral thrombosis and embolism. Stroke 21(12):1663-1667

15. Yi X, Lin J, Han Z, Zhou X, Wang X, Lin J (2012) The incidence of venous thromboembolism following stroke and its risk factors in eastern China. J Thromb Thrombolysis 34(2):269-275

16. Lyman GH, Khorana AA (2009) Cancer, clots and consensus: new understanding of an old problem. J Clin Oncol 27(29): $4821-4826$

17. Ay C, Vormittag R, Dunkler D, Simanek R, Chiriac AL, Drach J et al (2009) D-Dimer and prothrombin fragment $1+2$ predict venous thromboembolism in patients with cancer: results from the Vienna Cancer and Thrombosis Study. J Clin Oncol 27(25):4124-4129

18. Arpaia G, Carpenedo M, Verga M, Mastrogiacomo O, Fagnani D, Lanfredini $\mathrm{M}$ et al (2009) D-Dimer before chemotherapy might predict venous thromboembolism. Blood Coagul Fibrinolysis 20(3):170-175

19. Stender MT, Frokjaer JB, Larsen TB, Lundbye-Christensen S, Thorlacius-Ussing O (2009) Preoperative plasma D-dimer is a predictor of postoperative deep venous thrombosis in colorectal cancer patients: a clinical, prospective cohort study with one-year follow-up. Dis Colon Rectum 52(3):446-451

20. Yang Y, Zhou Z, Niu XM, Li ZM, Chen ZW, Jian H et al (2012) Clinical analysis of postoperative venous thromboembolism risk factors in lung cancer patients. J Surg Oncol 106(6):736-741

21. Ferroni P, Martini F, Portarena I, Massimiani G, Riondino S, La Farina F et al (2012) Novel high-sensitive D-dimer determination predicts chemotherapy-associated venous thromboembolism in intermediate risk lung cancer patients. Clin Lung cancer 13(6):482-487

22. Wu X, Xue X, Tang J, Cheng X, Tian W, Jiang R et al (2013) Evaluation of risk factors for venous thromboembolism in
Chinese women with epithelial ovarian cancer. Int J Gynecol Cancer 23(1):65-72

23. Kodama J, Seki N, Masahiro S, Kusumoto T, Nakamura K, Hongo A et al (2010) D-Dimer level as a risk factor for postoperative venous thromboembolism in Japanese women with gynecologic cancer. Ann Oncol 21(8):1651-1656

24. Palareti G, Legnani C, Cosmi B, Guazzaloca G, Pancani C, Coccheri S (2002) Risk of venous thromboembolism recurrence: high negative predictive value of D-dimer performed after oral anticoagulation is stopped. Thromb Haemost 87(1):7-12

25. Eichinger S, Minar E, Bialonczyk C, Hirschl M, Quehenberger P, Schneider B et al (2003) D-Dimer levels and risk of recurrent venous thromboembolism. JAMA 290(8):1071-1074

26. Palareti G, Legnani C, Cosmi B, Valdre L, Lunghi B, Bernardi F et al (2003) Predictive value of D-dimer test for recurrent venous thromboembolism after anticoagulation withdrawal in subjects with a previous idiopathic event and in carriers of congenital thrombophilia. Circulation 108(3):313-318

27. Verhovsek M, Douketis JD, Yi Q, Shrivastava S, Tait RC, Baglin $\mathrm{T}$, et al. Systematic review: D-dimer to predict recurrent disease after stopping anticoagulant therapy for unprovoked venous thromboembolism. Ann Intern Med. 2008;149(7):481-90, w94

28. Douketis J, Tosetto A, Marcucci M, Baglin T, Cushman M, Eichinger S et al (2010) Patient-level meta-analysis: effect of measurement timing, threshold, and patient age on ability of Ddimer testing to assess recurrence risk after unprovoked venous thromboembolism. Ann Intern Med 153(8):523-531

29. Becattini C, Lignani A, Masotti L, Forte MB, Agnelli G (2012) DDimer for risk stratification in patients with acute pulmonary embolism. J Thromb Thrombolysis 33(1):48-57

30. Squizzato A, Ageno W, Finazzi S, Mera V, Romualdi E, Bossi A et al (2006) D-Dimer is not a long-term prognostic marker following acute cerebral ischemia. Blood Coagul Fibrinolysis Int $\mathrm{J}$ Haemost Thromb 17(4):303-306

31. Di Napoli M, Papa F (2002) Inflammation, hemostatic markers, and antithrombotic agents in relation to long-term risk of new cardiovascular events in first-ever ischemic stroke patients. Stroke 33(7):1763-1771

32. Feinberg WM, Erickson LP, Bruck D, Kittelson J (1996) Hemostatic markers in acute ischemic stroke. Association with stroke type, severity, and outcome. Stroke 27(8):1296-1300

33. Knowlson L, Bacchu S, Paneesha S, McManus A, Randall K, Rose P (2010) Elevated D-dimers are also a marker of underlying malignancy and increased mortality in the absence of venous thromboembolism. J Clin Pathol 63(9):818-822

34. Ay C, Dunkler D, Pirker R, Thaler J, Quehenberger P, Wagner O et al (2012) High D-dimer levels are associated with poor prognosis in cancer patients. Haematologica 97(8):1158-1164

35. Ma X, Li Y, Zhang J, Huang J, Liu L (2013) Prognostic role of Ddimer in patients with lung cancer: a meta-analysis. Tumour Biol 35:2103-2109

36. Zorlu A, Yilmaz MB, Yucel H, Bektasoglu G, Refiker Ege M, Tandogan I (2012) Increased D-dimer levels predict cardiovascular mortality in patients with systolic heart failure. J Thromb Thrombolysis 33(4):322-328

37. Morange PE, Bickel C, Nicaud V, Schnabel R, Rupprecht HJ, Peetz D et al (2006) Haemostatic factors and the risk of cardiovascular death in patients with coronary artery disease: the AtheroGene study. Arterioscler Thromb Vasc Biol 26(12):27932799

38. Wen D, Du X, Dong JZ, Zhou XL, Ma CS (2013) Value of Ddimer and $\mathrm{C}$ reactive protein in predicting inhospital death in acute aortic dissection. Heart (British Cardiac Society). 99(16):1192-1197

39. Ohlmann P, Faure A, Morel O, Petit H, Kabbaj H, Meyer N et al (2006) Diagnostic and prognostic value of circulating D-dimers in 
patients with acute aortic dissection. Crit Care Med 34(5): 1358-1364

40. Querol-Ribelles JM, Tenias JM, Grau E, Querol-Borras JM, Climent JL, Gomez E et al (2004) Plasma D-dimer levels correlate with outcomes in patients with community-acquired pneumonia. Chest 126(4):1087-1092

41. Shilon Y, Shitrit AB, Rudensky B, Yinnon AM, Margalit M, Sulkes $\mathbf{J}$ et al (2003) A rapid quantitative D-dimer assay at admission correlates with the severity of community acquired pneumonia. Blood Coagul Fibrinolysis 14(8):745-748

42. Rodelo JR, De la Rosa G, Valencia ML, Ospina S, Arango CM, Gomez CI et al (2012) D-Dimer is a significant prognostic factor in patients with suspected infection and sepsis. Am J Emerg Med 30(9):1991-1999

43. Di Castelnuovo A, de Curtis A, Costanzo S, Persichillo M, Olivieri M, Zito F et al (2013) Association of D-dimer levels with all-cause mortality in a healthy adult population: findings from the MOLI-SANI study. Haematologica 98(9):1476-1480

44. Aujesky D, Roy PM, Guy M, Cornuz J, Sanchez O, Perrier A (2006) Prognostic value of D-dimer in patients with pulmonary embolism. Thromb Haemost 96(4):478-482

45. Righini M, Goehring C, Bounameaux H, Perrier A (2000) Effects of age on the performance of common diagnostic tests for pulmonary embolism. Am J Med 109(5):357-361
46. Lip GY, Lip PL, Zarifis J, Watson RD, Bareford D, Lowe GD et al (1996) Fibrin D-dimer and beta-thromboglobulin as markers of thrombogenesis and platelet activation in atrial fibrillation. Effects of introducing ultra-low-dose warfarin and aspirin. Circulation 94(3):425-431

47. Unlu Y, Karapolat S, Karaca Y, Kiziltunc A (2006) Comparison of levels of inflammatory markers and hemostatic factors in the patients with and without peripheral arterial disease. Thromb Res 117(4):357-364

48. Lindner G, Funk GC, Pfortmueller CA, Leichtle AB, Fiedler GM, Schwarz C et al (2014) D-Dimer to rule out pulmonary embolism in renal insufficiency. Am J Med 127(4):343-347

49. Shlipak MG, Fried LF, Crump C, Bleyer AJ, Manolio TA, Tracy RP et al (2003) Elevations of inflammatory and procoagulant biomarkers in elderly persons with renal insufficiency. Circulation 107(1):87-92

50. Torn M, Bollen WL, van der Meer FJ, van der Wall EE, Rosendaal FR (2005) Risks of oral anticoagulant therapy with increasing age. Arch Intern Med 165(13):1527-1532

51. Capodanno D, Angiolillo DJ (2012) Antithrombotic therapy in patients with chronic kidney disease. Circulation 125(21): 2649-2661 